Voix et Images

volxetimages

\title{
André Belleau, Y a-t-il un intellectuel dans la salle? Essais
}

\section{Robert Major}

Volume 11, numéro 1, automne 1985

\section{Naïm Kattan}

URI : https://id.erudit.org/iderudit/200543ar

DOI : https://doi.org/10.7202/200543ar

Aller au sommaire du numéro

Éditeur(s)

Université du Québec à Montréal

ISSN

0318-9201 (imprimé)

1705-933X (numérique)

Découvrir la revue

Citer cet article

Major, R. (1985). André Belleau, Y a-t-il un intellectuel dans la salle? Essais. Voix et Images, 11(1), 114-118. https://doi.org/10.7202/200543ar d'utilisation que vous pouvez consulter en ligne.

https://apropos.erudit.org/fr/usagers/politique-dutilisation/ 


\title{
André Belleau, $Y$ a-t-il un intellectuel dans la salle?Essais,
}

\author{
par Robert Major, Université d'Ottawa
}

La seule réaction convenable face à un essayiste étant de lui faire écho, de répercuter sa parole, selon Robert Vigneault (Voix et images, hiver 1983), je me vois frappé, dès l'abord, d'un sérieux empêchement. Il faudrait être doué d'une parole aussi personnelle que celle d'André Belleau ' pour lui faire écho et pour prolonger sa voix dans de nouvelles directions. Avoir une voix aussi timbrée, riche d'une pratique aussi diversifiée des hommes, des ouvres et de la vie... Me voilà réduit au silence!

Mais me rappelant fort opportunément qu'un écho est bêtement un obstacle, à l'origine, et une caricature du son initial, dans ses effets, je me sens, sinon ragaillardi, du moins validé (en quelque sorte!), et j'attaque. Et d'ailleurs, Robert Vigneault fait sans doute la partie plutôt belle aux essayistes, leur donnant tout le vécu pour province et les idées pour sujet, mais affirmant du même coup que leur discours est indiscutable et irrécusable, du seul ressort d'un autre essayiste. Vieille tentation de tuer le critique, mais d'une riche prospective, cette fois. Et si la seule réaction valable à un roman était, en effet, un autre roman; la seule réponse à un sonnet, une ballade... On se prend à rêver. Et à trembler pour son gagne-pain.

Les essayistes revendiquent. Tout, évidemment, selon l'humeur du moment ou les événements de l'heure; de l'indépendance du Québec au silence de Victor-Lévy Beaulieu; mais surtout, semble-t-il depuis quelque temps, leur place au soleil. Je crois que le coup d'envoi était donné ici par Jean Marcel en 11966 (Livres et auteurs canadiens 1965): dans nos lettres... l'essai l'emporte de beaucoup, quant au nombre, sur le roman et la poésie; en qualité, il n'est pas si loin de déclasser l'un et l'autre. À défaut d'une reconnaissance publique et de l'immortalité littéraire, que du moins on fasse aux essayistes leur place au sein de l'institution, qu'auraient outrageusement accaparée les théoriciens du discours poétique!

On sent le mouvement du pendule. Autrefois, les œuvres sérieuses (la prose d'idées) étaient en latin, langue de l'immortalité. Aux amusements des illettrés, la langue vernaculaire et ses ouvres. Et puis est venue l'éclipse (de quelques siècles!). Mais maintenant, le mouvement inverse s'accélère. On sent que la boucle sera bouclée, comme toujours plus rapidement ici qu'ailleurs, tellement nous nous sentons obligés de brûler les étapes pour nous faire pardonner d'être partis en retard. En peu de temps, nous sommes passés d'une méconnaissance de l'essai, d'une ignorance de ses codes et d'une dévalorisation de sa pratique (l'essayiste: romancier ou poète sans imagination), à une survalorisation du genre et de ses praticiens québéçois. Mais cette exaltation de l'essai entraînera sans doute bientôt un retour du pendule. Déjà elle ne laisse pas d'agacer ou de faire sourire. Car ces nombreuses protestations entraînent souvent l'effet contraire. À trop se faire dire que l'essayiste est un écrivain authentique, et peut-être même l'incarnation exemplaire de l'homme de plume, que son écriture n'a rien à envier à 
celle des romanciers et des poètes ou (Belleau) que la formation d'un essayiste exige beaucoup plus de temps que celle d'un poète ou d'un romancier (la durée ou la rigueur d'un apprentissage étant sans doute - le glissement s'effectue facilement - fonction de la noblesse et de l'importance de l'art pratiqué), le lecteur ordinaire en vient peu à peu à douter. The lady doth protest too much, methinks. Que l'essai occupe les premiers rangs du peloton ne correspond guère à sa propre expérience de lecture, ni à celle qu'il constate autour de lui (sauf en milieu universitaire où les exigences de participation à la rumeur publique, ou les simples règles de la politesse, faussent le libre jeu de la consommation...). Les Américains, qui ne s'y trompent pas, ne lui donnent pas cette place de tête dans leurs Great Books of the World ou autres listes de chefs-d'œuvre de tous les temps et de tous les pays. Quels sont les livres qui ont compté et qui comptent toujours, qui ont façonné notre univers? Chacun a sa liste; mais à côté des Essais de Montaigne ou des Pensées de Pascal, combien de pièces, de poèmes, de romans...

Dans sa "Petite essayistique", qui est en quelque sorte l'introduction de son très beau livre d'essais, André Belleau s'en prend assez vivement à la distinction désuète et quétaine entre les créateurs (romanciers et poètes) et les essayistes. Il a sans doute raison, pour la production récente, l'essai envahissant le roman et le roman, l'essai, et chacun racontant avec force mots l'aventure de l'écriture qu'il a vécue. Mais le vulgaire périgourdin que parle le lecteur moyen sait que les véritables aventuriers sont monnaie plus rare. Les essayistes revendiquent maintenant l'égalité avec les écrivains patentés (romanciers, poètes, dramaturges). Autrefois ils affichaient sans hésitation leur supériorité sur ces amuseurs publics. Je songe ici à cet autre essai (ou est-ce un conte?) de Washington Irving: The Mutability of Literature. A Colloquy in Westminster Abbey, où Irving imagine une conversation avec un antique in quarto dans les profondeurs de la bibliothèque abbatiale. Celui-ci, après s'être amèrement plaint d'avoir été négligé pendant des siècles, après s'être informé de ses contemporains, essayistes de savoir et d'intelligence dont on prédisait l'immortalité, est tout surpris d'apprendre que cet ignare de Shakespeare, a poor, half-educated varlet, that knew little of Latin, and nothing of Greek, est non seulement le plus connu des écrivains de son époque, mais aussi celui qui assure la survie des autres, giving duration to many an indifferent author, ainsi que l'assure le narrateur, merely from having flourished in his vicinity.

Here the little quarto began to heave his sides and chuckle, until at length he broke out in a plethoric fit of laughter that had well - nigh choked him, by reason of his excessive corpulency. "Mighty well!" cried he, as soon as he could recover breath; "mighty well! and so you would persuade me that the literature of an age is to be perpetuated by a vagabond deer-stealer! by a man without learning! by a poet, forsootha poet!" And here he wheezed forth another fit of laughter.

On aura beau dire, on aura beau s'en offusquer ou même s'en gausser, comme cet impertinent in quarto, on aura beau reconnaître que l'essai est du 
côté du savoir, de la culture et de l'intelligence (l'essai, invention littéraire de la vie pensée de l'homme, écrit encore R. Vigneault), toutes choses estimables bien entendu, ce genre, malgré qu'on en ait, reste un genre dérivé et l'essayiste n'occupera pas de sitôt, semble-t-il, les premiers rangs au panthéon des lettres. Art transitoire, art réactif, dépendant et secondaire (risquons le mot!) et qui n'a que rarement l'importance des autres genres: ceux qui, toujours selon R. Vigneault, construisent un monde autour de médiations symboliques.

Voilà! Ce moment d'humeur passé, qui risque de m'attirer des regards apitoyés, je précise tout de go que s'il a été provoqué par les réflexions sur l'essai dans le recueil d'essais d'André Belleau («Petite essayistique», "Approches et situation de l'essai québécois"), il ne préjuge en rien l'importance de ce livre et le plaisir que j'ai eu à lire son recueil. Et même ces essais sur l'essai, extrêmement stimulants et qui posent en termes savants mais clairs l'enjeu institutionnel de l'essai. D'ailleurs, pour dissiper toute ambiguité, il faut préciser que si Belleau valorise l'essai, ce n'est guère, à mon avis, par vain plaisir de prêcher pour sa chapelle. La lecture de son livre me laisse supposer qu'il serait incapable d'un tel calcul. Cette valorisation ne me semble plutôt qu'un des effets de l'engagement fondamental de l'auteur, qui traverse ce livre comme un leitmotiv: sa passion d'intelligence. Mais d'une intelligence bien particulière: concrète, matérielle, qui cherche l'enracinement dans les signes ambiants, qui sait dire shed ou smart ou quétaine quand il le faut mais peut aussi manier sans affectation les plus récents discours critiques pour éclairer son propos. Qui a en horreur toutes les formes d'obscurantisme, autant la démagogique (P.-E. Trudeau, V.-L. Beaulieu, Léandre Bergeron...) que la jargonneuse (l'oripeau de scientificité et la caution idéologique dont se drape l'enseignement de la littérature, par exemple). André Belleau, en effet, n'est pás loin de penser qu'il y a une idéologie québécoise dont le trait marquant est un anti-intellectualisme viscéral (même chez les intellectuels!) et contre laquelle doivent réagir toutes les personnes d'intelligence et de culture.

Mais attention! Intelligence et culture ne signifient pas snobisme et pédanterie. Et pour rappeler le vulgaire périgourdin, Belleau n'a rien du lettresférit, du frappé des lettres. Il distingue nettement (dans sa pratique autant qu'en théorie) discours savant et langage des salons, le deuxième seul étant exclusif. Il n'est guère indifférent, d'ailleurs, que son recueil se termine par un groupe d'essais consacrés à Rabelais, Rabelais qui se moquait des pédants et des marmonneurs, qui refusait le cloisonnement des langues et dont l'univers carnavalesque est une glorification du multiple. Mais qui est foncièrement savant aussi, véritable encyclopédie du savoir de son temps. Des affinités sont certainement à l'œuvre ici. L'entreprise de Belleau ne saurait être qualifiée de gargantuesque, certes, mais un type particulier d'intelligence, profondément matérielle, qui sait tirer son profit du tremblement d'une feuille, du cri d'un cocher, de la réaction d'un consommateur, tout autant que de l'audition de Brahms ou de la lecture d'un mathématicien, le rapproche indubitablement de son auteur d'élection. Décaper les mots, serrer de près la réalité, croquer dans la vie, sans les aspects truculents que 
nous associons à la verve rabelaisienne, mais avec un égal plaisir, semble-t-il, et un certain ton qui ne doit rien au gigantisme sauf peut-être à ce qu'il a d'essentiel: sa bienveillance et sa bonhomie.

Car la bienveillance me semble le ton dominant du recueil. À ma surprise, d'ailleurs, car au cours des années et d'une lecture partielle et éclatée de ces textes (la plupart parus dans Liberté et Voix et images depuis vingt ans), je m'étais construit la figure d'un Belleau plus critique, plus pamphlétaire, plus ironique. Surprise qui confirme la justesse de la réflexion de François Ricard (Études françaises, oct. 1977) à l'effet que la republication d'essais courts en recueil les révèle littéralement, les éclaire de la lumière organique qui y était déjà mais que la première publication, événementielle et fragmentée, cachait. Une passion d'intelligence et une bienveillance, telle est la nécessité intérieure de ce recueil. Et s'il y a ironie chez Belleau, il s'agit de cette ironie supérieure de l'essayiste dont parlait Lukacs (Luckacs qu'aime aussi Belleau), ironie qui fait croire qu'il n'est question que de livres et de tableaux alors que l'essayiste parle des questions ultimes de la vie (L'Ầme et les formes). Belleau signale, rapproche, décrit, interroge et réfléchit. Il ne raille pas. Il a ses bêtes noires, certes, au Devoir ou à Radio-Canada, du côté des formalistes ou des joualisants, mais le respect des personnes est sans failles, et l'humour ou le regard amusé ne glisse que très rarement au brocard. Belleau, je le dis avec admiration et sans connaître la personne - le Belleau dont je parle est le narrateur de ces essais -, est savant de la première des sciences, celle qui permet d'avaler les autres sans dommage: la science de la bonté. (On aura deviné que j'ai relu mon Montaigne! En effet, ce livre m'a donné le goût de relire bien des choses, autre signe de sa réussite.)

J'ai donc passé de belles heures en sa compagnie, à écouter cette voix intelligente et chaleureuse; et je suis reconnaissant à ses amis de l'avoir poussé à rapailler ses essais (ainsi qu'à Belleau d'avoir répondu à leurs sollicitations). J'ai quelquefois regretté ce qu'on pourrait appeler sa rhétorique de l'humble réserve (il conviendrait, pour compléter ces éléments assez sommairement rassemblés... dans un texte hélas! insuffisamment développé... ce ne sont là que quelques remarques... je propose ma lecture comme essentiellement provisoire... je ne sais que conclure... ces brèves remarques ne se proposaient... les quelques questions que j'ai pu soulever...), rhétorique un peu agaçante et d'ailleurs inutile car Belleau sait mieux que quiconque que l'essai est par définition pensée inachevée et asystématique (Ricard). Il me semble à son meilleur quand il n'essaie pas de faire long (paradoxalement, chaque fois qu'il fait un peu plus long, il s'excuse de ne pas faire encore plus long!). C'est pourquoi les essais écrits pour Liberté me semblent plus réussis que ceux (plus officiellement critiques ou professoraux) écrits pour Voix et images ou en vue de communications diverses; façon de confirmer ce que luimême dit du rôle discursif de cette revue exemplaire qu'est Liberté. Quand Belleau fait court, il fait vraiment "autre chose», selon sa propre formule pour désigner la nouvelle. 
Terminons par le début, par un mot sur le titre, premier contact avec un texte, sa synecdoque et son embrayeur. Je n'aime guère celui-ci, dont je dois reconnaître l'efficacité, mais qui me semble inutilement racoleur, alors que la manière de Belleau n'est pas du tout de cet ordre. Son ton est au contraire serein, sage, équilibré, bon vivant mais exigeant. Je le trouve donc mal inspiré d'adopter - et même si c'est pour les subvertir - les mots du repoussoir (il s'agit de P.-E. Trudeau). Car avec les mots, vient le ton de ce dernier: gouailleur et agressif, on le sait assez!

1. André Belleau, Y a-t-il un intellectuel dans la salle? Essais, Editions Primeur, 1984, 206 p. 